\title{
Preemptive non-selective bronchial artery angioembolization to reduce recurrence rate of hemoptysis
}

\author{
Mohammad Sadegh Keshmiri, ${ }^{1}$ Shadi Shafaghi, ${ }^{1}$ Babak Sharif-Kashani, ${ }^{2}$ Ali Sadoughi, ${ }^{3}$ Fariba Ghorbani, \\ Farah Naghashzadeh, ${ }^{1}$ Atefeh Abedini ${ }^{5}$
}

${ }^{1}$ Lung Transplantation Research Center, National Research Institute of Tuberculosis and Lung Diseases (NRITLD), Shahid Beheshti University of Medical Sciences, Tehran, Iran

${ }^{2}$ Tobacco Prevention and Control Research Center, National Research Institute of Tuberculosis and Lung Diseases (NRITLD), Shahid Beheshti University of Medical Sciences, Tehran, Iran

${ }^{3}$ Divisions of Pulmonary and Critical Care, Albert Einstein College of Medicine, New York, USA

${ }^{4}$ Tracheal Diseases Research Center, National Research Institute of Tuberculosis and Lung Diseases, Shaheed Beheshti

University of Medical Sciences, Tehran, Iran

${ }^{5}$ Chronic Respiratory Diseases Research Center, National Research Institute of Tuberculosis and Lung Diseases (NRITLD), Shahid Beheshti University of Medical Sciences, Tehran, Iran

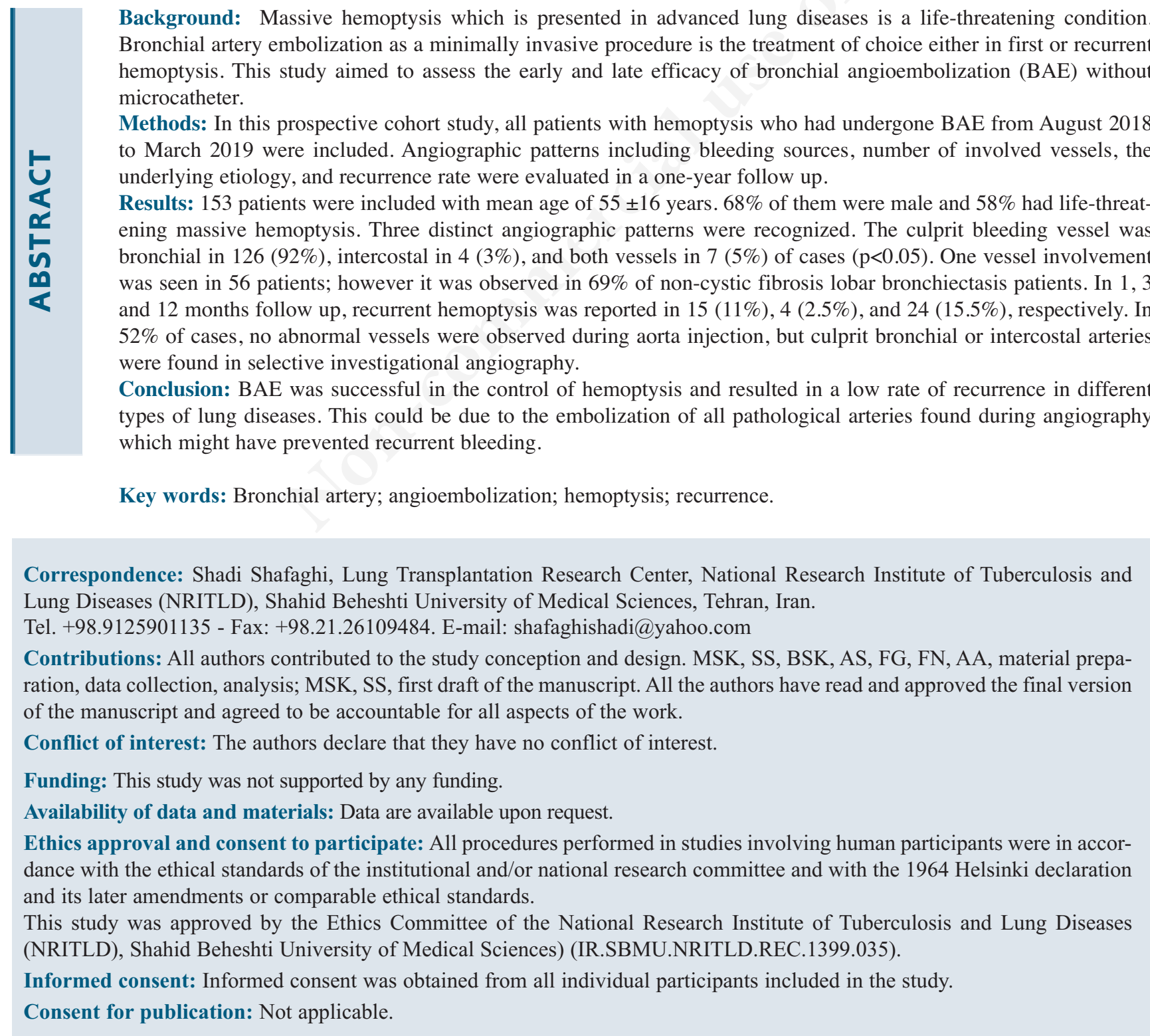




\section{Introduction}

Hemoptysis is defined as coughing up blood or bloody sputum from the lower respiratory tract, including the trachea and the bronchi [1]. It is a vascular complication caused by a number of lung diseases, including but not limited to bronchiectasis, tuberculosis, and congenital anomalies of the pulmonary arteries [2]. Various definitions have been proposed for massive hemoptysis. Although some studies considered different volume of the blood excreted over 24 hours to be as massive hemoptysis, it seems the condition of life-threatening hemoptysis is not always related to the amount of blood, since it depends on the clinical condition of the patient (dyspnea, $\mathrm{O}_{2}$ desaturation, agitation state, hemodynamic instability) [3]. Relying on only conservative management will result in the death of $50 \%$ to $100 \%$ of patients [4]. Asphyxia accounts for $80 \%$ of mortality and morbidities in massive hemoptysis $[5,6]$. The most common causes of massive hemoptysis are: tuberculosis in developing countries and primary lung cancer, bronchiectasis, cystic fibrosis (CF), aspergillosis, and sarcoidosis in developed countries [5]. In case of hemoptysis, emergency treatment should be performed by a team consisting of a pulmonologist, a thorax surgeon, a radiologist, an anesthesiologist, and a cardiologist. Airway protection is performed using a single lumen tube along with a balloon blocker as the recommended method or a double lumens trachea tube. If possible, diagnostic tests are performed to determine the location and type of lesion [7].

After resuscitation and airway protection, bronchial artery embolization as a minimally invasive procedure is the treatment of choice in critically ill patients with life-threatening hemoptysis [8].
Bronchial vascular embolization has been introduced since 1970 to save the patients' life with high efficacy and low side effects [9]. To embolize the bronchial artery, angiography is performed to inject an embolic agent into the abnormal bronchial artery in order to completely block the bleeding vessel [10-13]. Among the embolic agents, the following can be mentioned: polyvinyl alcohol (PVA), spherical and non-spherical particles, gelatin, sponge pledgets, PVA hydrogel, tris-acryl gelatin microspheres, acrylic glue, and steel coil. This procedure is the first-line treatment of lifethreatening massive hemoptysis, especially in patients who are unable to undergo surgery due to an underlying disease [3]. This procedure is recommended only in highly experienced centers of pulmonary diseases that are active in the field of pulmonary angioembolization. In this study, we presented the data regarding bronchial angioembolization (BAE) in a large referral center and assessed its outcomes in the treatment of hemoptysis in a wide variety of etiologies.

\section{Methods}

Patients medical records including demographic data, underlying diseases, number of embolized vessels, fluoroscopy findings, side and origin of involved vessels, and complications of all patients with hemoptysis who have undergone bronchial angioembolization from August 2018 to March 2019, were evacuated. The patient's general condition, as well as the status of hemoptysis recurrence, patient survival, and other treatments related to hemoptysis, were obtained via phone contact and the relevant question-

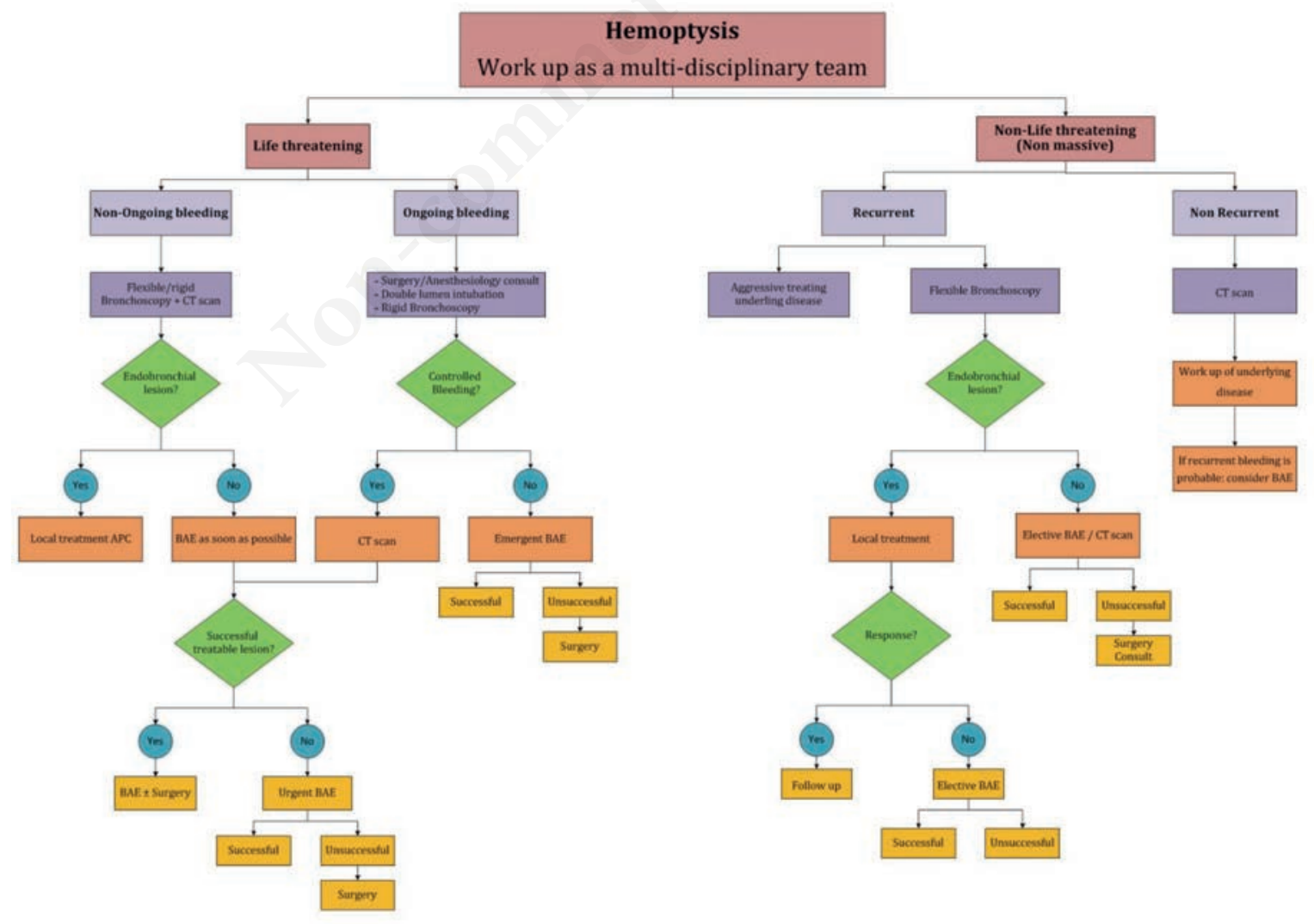

Figure 1. Approach to hemoptysis and the possibility of bronchial angioembolization. APC, argon plasma coagulation; BAE, bronchial angioembolization. 
naire was completed. The approach to hemoptysis in our center has shown in Figure 1. In case of unsuccessful BAE in endobronchial lesions, further approach was a repeat bronchoscopy and local treatment using electrocautery, argon plasma coagulation (APC), tranexamic acid inhalation. After prep. and drep. through the right femoral artery, arterial access was obtained, then the $5 \mathrm{~F}$ pigtail catheter (Cordis Co., Vaughan, CA, USA) was inserted into the descending aorta and aortography was performed. In most of the aortograms, bronchial and intercostal arteries were observed, which were used as guides to find abnormal blood vessels (torturous, aneurysm, fistula, and ectasia). When bronchial or intercostal arteries were not visible, an attempt was made to find abnormal arteries through an investigative approach. Then, using the 5F C1 (Cobra) catheter (Merit Medical Systems, Inc., South Jordan, UT, USA) or the Tiger 5F catheter (Merit Medical Systems, Inc.), angiography of the bronchial and intercostal arteries was performed selectively, and in case of abnormalities such as vascular ectasia, vascular torsion, fistula, or hypervascularity embolization was performed selectively using PVA drug with a size of 500 to 700 microns (Figure 2 A,B). Additionally, in "suspicious" or spinal cord branch artery involvement, embolization was performed by a super-selective method using the Embo catheter $2.8 \mathrm{~F}$ (Merit Medical Systems, Inc.) (Figure 2 C,D). "Suspicious" branches were considered as vascular branches supply to the spinal cord, eyes, or brain. Embolization of these vessels can lead to serious complications including spinal cord ischemia or injury leading to paraplegia, paraparesis, blindness, or cerebral ischemia. Statistical analysis was performed employing SPSS 21. Quantitative variables were expressed as means $\pm \mathrm{SD}$, and qualitative data were expressed as proportions. To compare different study groups, ANOVA followed by post-hoc test (Scheffe) was used after normal-distribution-confirmation regarding Kolmogorov-Smirnov test. Also, Chi-square for non-parametric samples or categorical variables were considered with confidence interval (CI) of $95 \%$ and a significant level of 0.05 .

\section{Results}

A total of 153 patients were enrolled in the study, with mean age of $55 \pm 16$ years and $68 \%$ male. Meanwhile, $87 \%$ of patients experienced hemoptysis for the first time and $58 \%$ had life-threatening massive hemoptysis. In only $3 \%$ of cases, no embolization was performed due to the lack of any pathologic or embolizable vessel.

\section{Underlying diseases}

Table 1 depicts the most common causes of hemoptysis.

\section{Side of involved vessels}

Lung CT scans showed bilateral lesions in 80 (52\%), unilateral lesions in the right lung in $40(26 \%)$, and unilateral lesions in the left lung in $22(14 \%)$ of the cases. In $8 \%$ of cases, no lesions were seen on CT scan of the lungs. In $54 \%$ of angioembolization cases, the lung involvement was bilateral and $10.8 \%$ bifurcated vessels were observed. $82 \%$ of abnormal blood vessels had fistulas. In CF bronchiectasis, non-CF disseminated bronchiectasis, tuberculosis, and cancer the most common feature is bilateral involvement of lungs $(83 \%, 46 \%, 61 \%$, and $60 \%$, respectively) (Table 2).

\section{Origin of involved vessels}

In 128 patients $(93 \%)$ the lesions were observed in the bronchial vessels, in 4 patients $(2 \%)$ the lesions were in the intercostal vessels, and in 7 patients $(5 \%)$ the lesions were in both the bronchial and intercostal vessels. The pattern of involved vessels was significantly different in disease categories $(p<0.05)$. Although in CF-bronchiectasis and Wegener and anthracosis, bronchial arteries were the only involved vessels, angioembolization demonstrated the mixed pattern of bronchial or intercostal or bronchial-intercostal vessel abnormality in non-CF lobar/disseminated bronchiectasis and tuberculosis and cancer (Figure 3).

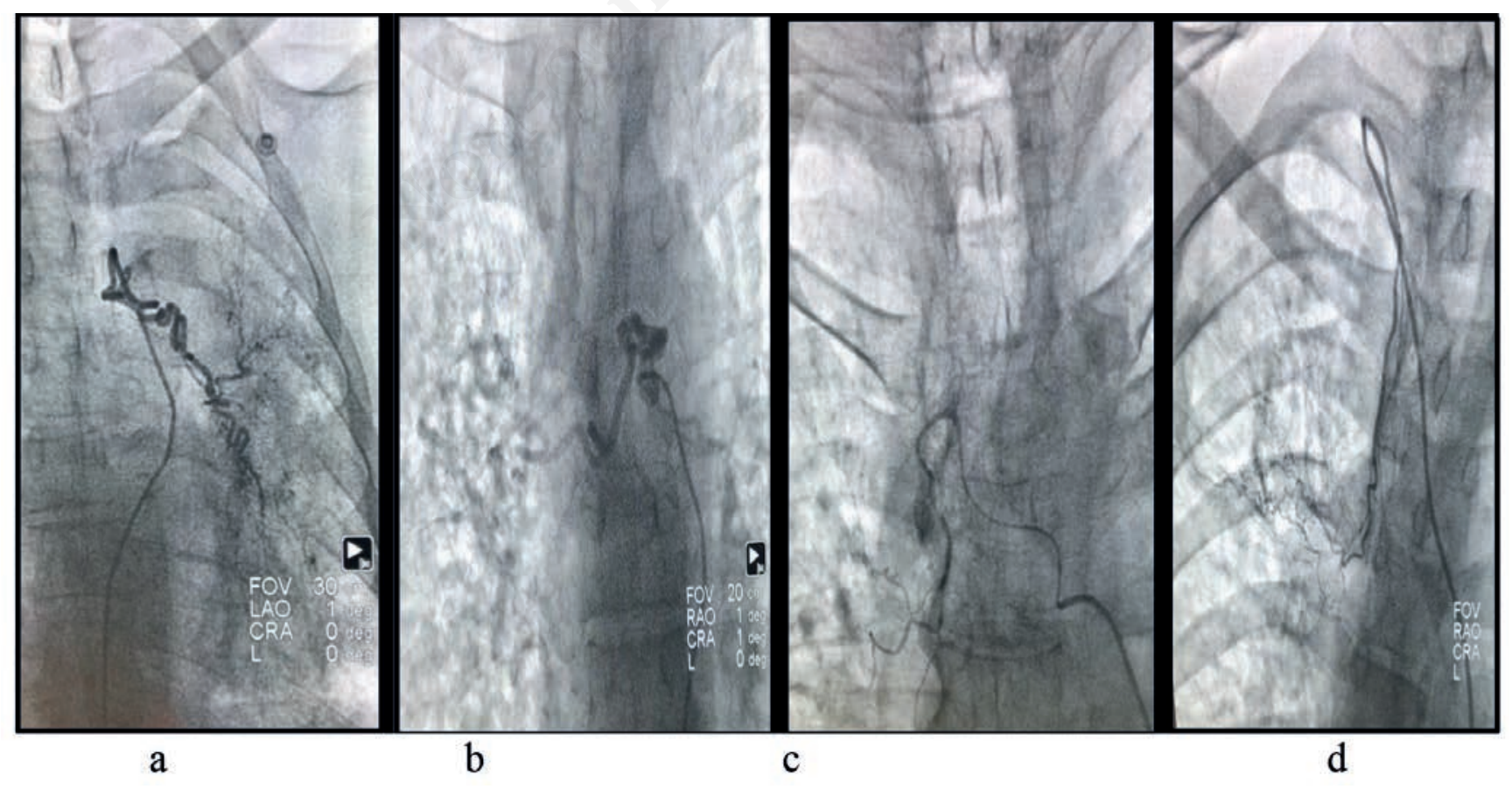

Figure 2. a) Left bronchial artery angioembolization. b) Right bronchial artery angioembolization. c) Super selective bronchial artery angioembolization. d) Super selective right subclavian artery angioembolization. 


\section{Number of involved vessels}

Generally, in $38 \%$ of cases, lesions were observed in one vessel, $36 \%$ in two vessels, $18 \%$ in three vessels, $3 \%$ in four vessels, and $2 \%$ more than four vessels were involved. Moreover, in nonCF lobar bronchiectasis one vessel involvement is the most feature $(69 \%)$, meanwhile in disseminated bronchiectasis one, two and three-vessel involvement has $45 \%, 25 \%$, and $22 \%$ proportion of abnormalities. In tuberculosis, $27 \%$ of patients have three or more involved vessels (Table 3).

While in $48 \%$ of cases, abnormal blood vessels were observed when the contrast agent was injected into the descending aorta, in the remaining $52 \%$ of cases, no abnormal vessels were observed during aorta injection. Bronchial or intercostal arteries were found selectively through investigative angiography. Seventeen patients (11\%) underwent super-selective angioembolization due to spinal branches seen during angioembolization.

\section{Fluoroscopy findings and zones of involved vessels}

We classified the lesions into 3 different zones based on their fluoroscopy appearance: zone 1 nearby the left bronchus, zone 2 above the left bronchus, and zone 3 below the left bronchus

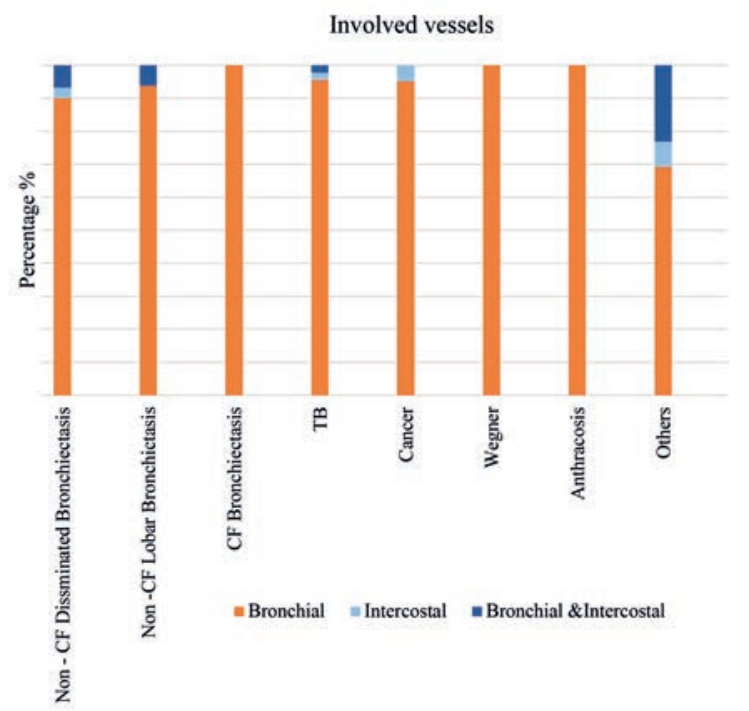

Figure 3. Involved vessels in different etiologies.

Table 1. The most common causes of hemoptysis and distribution of age and sex in different groups.

\begin{tabular}{lccc} 
Disease & Frequency (\%) & $\begin{array}{c}\text { Age (year) } \\
\text { Mean } \pm \text { SD }\end{array}$ & Male\%) \\
Lobar non-CF bronchiectasis & $19(12.7)$ & $47 \pm 17.3$ & 73.7 \\
Disseminated non-CF bronchiectasis & $33(21.6)$ & $57.85 \pm 14.58$ & 69.7 \\
Cystic fibrosis & $6(4)$ & $46.38 \pm 10.4$ & 33 \\
TB & $23(31.3)$ & $56.9 \pm 13.2$ & 59.6 \\
Cancer & $23(15.3)$ & $63 \pm 12.4$ & 78.3 \\
Wegner & $2(1.3)$ & $32.50 \pm 3.5$ & 100 \\
Anthracosis & $5(3.3)$ & $66.2 \pm 17.4$ & 80 \\
Others & $15(10)$ & $50.7 \pm 21.2$ & 66.7 \\
\hline
\end{tabular}

CF, cystic fibrosis.

Table 2. Lung involvement in hemoptysis patients.

\begin{tabular}{lcccccccc}
$\begin{array}{l}\text { Involved } \\
\text { region }\end{array}$ & $\begin{array}{c}\text { Disseminated } \\
\text { bronchiectasis }\end{array}$ & $\begin{array}{c}\text { Lobar } \\
\text { bronchiectasis }\end{array}$ & $\begin{array}{c}\text { CF } \\
\text { bronchiectasis }\end{array}$ & Tuberculosis & Cancer & Wegner & Anthracosis & Other \\
Right & $5(19.2 \%)$ & $5(35.7 \%)$ & $0(0.0 \%)$ & $8(20.5 \%)$ & $5(25 \%)$ & $0(0) \%$ & $3(60 \%)$ & $4(33.3 \%)$ \\
Left & $9(34.6 \%)$ & $4(28.60 \%)$ & $1(16.7 \%)$ & $7(17.9 \%)$ & $3(15 \%)$ & $1(5 \%)$ & $0(0) \%$ & $3(25 \%)$ \\
Bilateral & $12(46.2 \%)$ & $5(35.7 \%)$ & $5(83.3 \%)$ & $24(61.5 \%)$ & $12(60 \%)$ & $1(50 \%)$ & $2(40 \%)$ & $5(41.7 \%)$ \\
Total & 26 & 14 & 6 & 39 & 20 & 2 & 5 & 12 \\
\hline
\end{tabular}

Table 3. Number of embolized vessels in different disease.

\begin{tabular}{|c|c|c|c|c|c|}
\hline \multirow[t]{2}{*}{ Disease } & \multicolumn{5}{|c|}{ Number of embolized vessel } \\
\hline & 1 & 2 & 3 & 4 & More than 4 \\
\hline Lobar non-CF bronchiectasis & $14(45.2 \%)$ & $8(25.8 \%)$ & $7(22.6 \%)$ & $1(3.2 \%)$ & $1(3.2 \%)$ \\
\hline Disseminated non-CF bronchiectasis & $11(68.8 \%)$ & $4(25.0 \%)$ & $1(6.3 \%)$ & $0(0.0 \%)$ & $0(0.0 \%)$ \\
\hline Cystic fibrosis & $1(16.7 \%)$ & $0(0.0 \%)$ & $3(50.0 \%)$ & $2(33.3 \%)$ & $0(0.0 \%)$ \\
\hline Tuberculosis & $13(28.9 \%)$ & $20(44.4 \%)$ & $10(22.2 \%)$ & $1(2.2 \%)$ & $1(2.2 \%)$ \\
\hline Cancer & $8(34.8 \%)$ & $13(56.5 \%)$ & $2(8.7 \%)$ & $0(0.0 \%)$ & $0(0.0 \%)$ \\
\hline Wegner & $1(50 \%)$ & $0(0.0 \%)$ & $1(50 \%)$ & $0(0.0 \%)$ & $0(0.0 \%)$ \\
\hline Anthracosis & $2(40 \%)$ & $2(40 \%)$ & $1(20 \%)$ & $0(0.0 \%)$ & $0(0.0 \%)$ \\
\hline Others & $6(42.9 \%)$ & $4(28.6 \%)$ & $2(14.3 \%)$ & $1(7.1 \%)$ & $1(7.1 \%)$ \\
\hline
\end{tabular}


(Figure 4). As the graph depicted, the most common site of involvement seen in fluoroscopy is nearby the left bronchus also called the hilar region of the lung (zone 1), and the next common site is above the left bronchus (zone 2).

\section{Complications}

Among angioembolization cases, 98\% were performed without any major complications. Ischemia and intercostal muscle pain in $0.5 \%$, pulmonary infarction in $1 \%$, and spinal complications in $0.5 \%$ of the patients were reported. However, esophagitis, head and neck complications, and inadvertent embolism in other organs were not observed in any of the patients. In the event of new-onset dyspnea, cough or any other sign attributable to allergic reaction to PVA use during BAE, $8 \mathrm{mg}$ dexamethasone was administered via intravenous route during angioembolization. It is considerable that dexamethasone was used only in $30(20 \%)$ patients. There was no significant difference in the outcome between the two groups who received or do not receive dexamethasone.

\section{Outcomes}

Bleeding recurrence was reported in 15 patients (11\%) after one month. One-month recurrence in the setting of one or two lesions occurred in 11 patients $(73.3 \%)$ and $4(26.7 \%)$ patients with more than 2 abnormal vessels. Recurrence was reported in $8.9 \%$ of patients with a single lesion, $11.3 \%$ with 2 lesions, and $66.7 \%$ with more than 4 vessels involved. There was no correlation between one-month recurrence and type of disease; $43 \%$ of one-month recurrence belonged to non-CF disseminated bronchiectasis. Bleeding recurrence after 3 months was reported in 3\% of patients. In 24 patients $(15 \%)$, bleeding recurrence occurred after one year, 14 cases of them $(58 \%)$ were treated with medication, 3 cases (12\%) underwent bronchoscopy, and no specific treatment was performed in 3 cases $(12 \%) .15$ cases $(62 \%$ of recurrence cases and in other words, $9 \%$ of all patients) underwent re-angioembolization. In patients with recurrent hemoptysis, $82 \%$ had one, $15 \%$ had two, $2 \%$ had three, and $1 \%$ had more than three recurrent hemoptysis episodes. Sixteen percent of all recurrences were in the form of massive hemoptysis. Out of 23 patients $(15 \%)$ who died within a year of embolization, only one was due to massive hemoptysis. Other causes of death were due to non-bleeding complications of the underlying disease, including cancer and bronchiectasis.

\section{Discussion}

Early and late results of non-selective bronchial angioembolization (BAE) without microcatheter revealed that this method as the first-line treatment in most patients with massive hemoptysis was successful to control bleeding and had low recurrence rate.

In this non-surgical minimally invasive treatment, during aortography, hemostasis of the bleeding vessel is attained using very fine particles or coils or gel foams [10-13]. Due to the high mortality rate of massive hemoptysis, taking emergent diagnostic and therapeutic measures by an experienced team consisting of a thorax surgeon, a pulmonologist, an anesthesiologist, radiologist or interventional cardiologist will help save the patient's life [7].

Numerous diseases may lead to massive hemoptysis. A study in Singapore by Peter et al. reported that tuberculosis was the leading cause of massive hemoptysis in $1 / 3$ of the cases [14]. In a study by Shigemura [15] in China from 2000 to 2005, 62 patients underwent angioembolization due to massive hemoptysis and the underlying causes included tuberculosis in $55 \%$, bronchiectasis in $23 \%$, mycetoma in $8 \%$, lung cancer in $6 \%$, and necrotizing pneumonia in $6 \%$ of the cases. In a study conducted by Knott et al. in South Africa, tuberculosis was responsible for $85 \%$ of massive hemoptysis cases [16]. In Austria, a cohort study [17] from 2000 to 2004 showed that the leading causes of massive hemoptysis were lung cancer (34\%) and then tuberculosis (23\%). Fartoukh [18] also reported that among 196 massive hemoptysis patients admitted to ICU in a hospital in Paris, $40 \%$ had bronchiectasis, $17 \%$ had lung
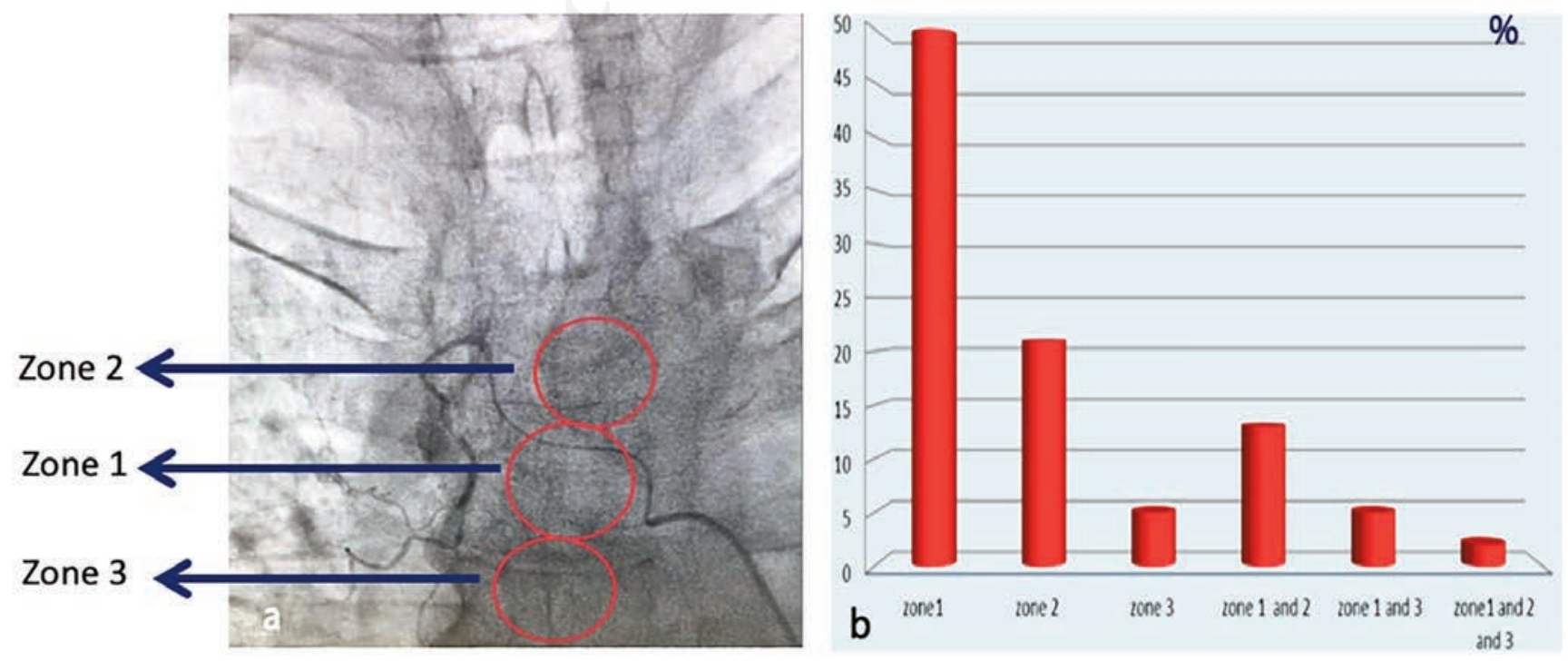

Involved zone

Figure 4. a) Fluoroscopy images of zone classification during bronchial artery angioembolization. b) Frequency of vascular involvement in different zones. Zone 1, nearby left bronchus; zone 2, above the left bronchus; zone 3, below the left bronchus. 
cancer, and $14 \%$ had active tuberculosis. The most common causes of massive hemoptysis in developing countries are tuberculosis, and in developed countries are primary lung cancer, bronchiectasis, cystic fibrosis, aspergillosis, and sarcoidosis [5]. A study in Iran demonstrated lung cancer, pulmonary hypertension, bronchiectasis, and tuberculosis as the most common etiology of hemoptysis $(63 \%, 63 \%, 31.7 \%, 25 \%$, respectively) [19]. In the present study, the most common disease leading to massive hemoptysis was tuberculosis (32\%). Diffuse non-cystic fibrosis bronchiectasis, cancer, lobar non-cystic fibrous bronchiectasis, and cystic fibrosis have been ranked next $(22 \%, 15 \%, 12 \%$, and $4 \%$, respectively).

In dealing with a patient with massive hemoptysis after taking a complete history (symptoms of active infection, recent surgery, taking anticoagulants or antiplatelet drugs, a history of malignancy, tuberculosis, underlying lung disease) and a thorough physical examination, it is necessary to stabilize the patient's hemodynamics first and then if possible, determine the exact bleeding site before proceeding to bronchial angioembolization [20].

Due to the low diagnostic power of chest X-ray [21] and bronchoscopy in determining the location of the lesion, which is $(48 \%)$ [22], the lesion site was determined using a spiral CT scan of the lungs. A prospective study of 606 patients showed that CT scan alone had $77 \%$ diagnostic power in determining the cause of bleeding, whereas CT scan along with bronchoscopy at the same time showed $83 \%$ diagnostic power [22]. Also in other studies conducted in 2001, the diagnostic power of a multi-detector CT in determining bleeding site was shown to be $63 \%$ to $100 \%$ [23,24]. These studies showed that in $94 \%$ of cases, the lesion site could be localized using CT scan, and in only $6 \%$ of cases, no lesion was observed in a spiral CT scan. Most of these patients with normal spiral CT scan had mild diffuse or lobar bronchiectasis detected in high resolution computed tomography (HRCT) or congenital vascular anomalies found in aortic angiography. A 94\% accordance of the lesion site in CT scan and angiography indicates that CT scan as a guide can be of great help, but the findings during angiography do not necessarily correspond to the findings of CT scan and the final decision regarding angioembolization should be made according to the vascular pattern observed during angiography. Injection of contrast agent in the descending aorta showed abnormal vessels in only $47 \%$ of the cases. Investigative angiography of the bronchial or intercostal arteries selectively found $52 \%$ of the abnormal vessels. CT angiogram was not done in every case, and the authors believe aortic angiography with selective bronchial and intercostal artery examination may detect abnormal small vessels that can be missed by CT angiogram. In cases where there is suspicion of aberrant spinal branches, or aberrant branches from left inter mammary artery (LIMA), right inter mammary artery (RIMA), or subclavian artery, embolization by a super-selective method using a microcatheter to occlude the culprit lesion only is preferred and will help prevent spinal nerve complications and or blockage of the healthy arterial [25] branches.

The patient may have lesions or abnormal bronchial arteries in both lungs seen in angiogram; however there is usually only one site that is actively bleeding (culprit lesion). The authors speculate that embolization of all pathological arteries found during angiography might have prevented possible future re-bleeding. The low recurrence rate of hemoptysis after one year in this center compared to current available data [26] indicates the efficiency of the strategies applied in this center. It suggests the efficacy of our approach in embolization of all pathological arteries observed during angiography and not just culprit arteries in decreasing the recurrence rate. One obstacle to this approach is time constraints due to possible patient clinical instability.

In $90 \%$ of massive hemoptysis cases, the bleeding component is in the bronchial circulatory system [4]. Knowledge of bronchial artery anatomy is essential in planning how to perform embolization in a patient $[4,27]$. On the other hand, non-bronchial arteries are also responsible for cases of massive hemoptysis and may cause recurrence of hemoptysis after successful embolization [4].

Knowledge of the bronchial artery anatomy, together with an understanding of the pathophysiologic features of massive hemoptysis, is essential for planning and performing BAE in affected patients. In our experience, the descending aorta view in fluoroscopy was divided into three zones: zone 1 is nearby the left bronchus; zone 2 is located above the left bronchus and zone 3 is below the left bronchus. This classification helped in detecting abnormal blood vessels during embolization. The most common affected area was is zone one, followed by zone two. In about $70 \%$ of cases, abnormal arteries originate from the aorta nearby and upper than the left bronchus. However, in some cases, multiple vessels were involved simultaneously from several zones.

Angioembolization of the bronchial artery, rigid and flexible bronchoscopy, and surgery have all been suggested as treatment options to control and treat bleeding in patients with massive hemoptysis. However, there is still no clear protocol for the role of each of these procedures and how to prioritize them [20]. Due to the high mortality rate of emergent surgery in patients with massive hemoptysis (about 40\%) [19], in this study, an attempt was made to postpone the surgery even if the patient was a candidate for thoracotomy surgery based on the findings of CT scan, and consider bronchial angioembolization beforehand if possible [28], in which case the probability of death was expected to be much lower $(7.1 \%$ to $18.2 \%)$ [4]. In 2018 , Springer et al. presented results of angioembolization of bronchial arteries in 30 patients who underwent 47 procedures from 2011 to 2017 [29]. In 95\% of patients, rapid success in controlling hemoptysis was achieved and $10 \%$ of patients had recurrence during the first 3 days, 4 of whom underwent BAE again and had no recurrence within 63 months. Eleven patients (36\%) had relapses requiring BAE [29]. Numerous causes, such as tuberculosis, aspergilloma, systemic non-bronchial accessory blood flow, or bronchopulmonary shunts have been identified as causes of recurrent bleeding after angioembolization [27-29]. Although the results of several studies have shown that the early success rate of this minimally invasive procedure in controlling severe bleeding is very high $(70 \%$ to $99 \%)$, the one-month recurrence rate of hemoptysis (58\%) is still considerable [26]. The finding of our study demonstrated one month, three-month, and one-year bleeding recurrence was $11 \%, 3 \%$, and $15 \%$ of patients, respectively. That is comparable with global results, likely due to technical and procedural differences used in our center as described below.

\section{Conclusion}

Preemptive angioembolization of abnormal but non-bleeding vessels during BAE in tuberculosis and bronchiectasis patients seems to prevent further recurrence. It may help decrease mortality due to the fact that the highest prevalence of hemoptysis is observed in these patients' population. Adapting super-selective methods using a microcatheter when there is suspicion of aberrant spinal branches or others coming from the internal mammary or subclavian artery is recommended in order to occlude the culprit vessel only and avoid complications.

\section{Acknowledgments}

We appreciate the cooperation of the Research Deputy of Masih Daneshvari Hospital in Tehran. 


\section{References}

1. Adachi T, Oki M, Saka H. Management considerations for the treatment of idiopathic massive hemoptysis with endobronchial occlusion combined with bronchial artery embolization. Intern Med 2016;55:173-7.

2. Albahhar M, Vo HA, Bowers H, Keshavamurthy J, Rotem E. Embolization of non-bronchial systemic artery (inferior phrenic) as a source of massive hemoptysis in a patient with mycobacterial bronchiectasis. Am Thorac Soc 2017;195: A1491.

3. Ibrahim WH. Massive haemoptysis: the definition should be revised. Eur Respir J 2008;32:1131-2.

4. Yoon W, Kim JK, Kim YH, Chung TW, Kang HK. Bronchial and nonbronchial systemic artery embolization for life-threatening hemoptysis: a comprehensive review. Radiographics 2002;22:1395-409.

5. Jean-Baptiste E. Clinical assessment and management of massive hemoptysis. Crit Care Med 2001;29:1098.

6. Xie M. Value of radiodensity determined by enhanced computed tomography for the differential diagnosis of lung masses. Iran J Radiol 2011;8:145.

7. Gupta A, Sands M, Chauhan NR. Massive hemoptysis in pulmonary infections: bronchial artery embolization. J Thorac Dis 2018;10:S3458.

8. O'Dell MC, Gill AE, Hawkins CM. Bronchial artery embolization for the treatment of acute hemoptysis. Tech Vasc Interv Radiol 2017;20:263-5.

9. Chun JY, Belli AM. Immediate and long-term outcomes of bronchial and non-bronchial systemic artery embolisation for the management of haemoptysis. Eur Radiol 2010;20:558-65.

10. White RI. Bronchial artery embolotherapy for control of acute hemoptysis: analysis of outcome. Chest 1999;115:912-5.

11. Tanaka N, Yamakado K, Murashima S, Takeda K, Matsumura $\mathrm{K}$, Nakagawa T, et al. Superselective bronchial artery embolization for hemoptysis with a coaxial microcatheter system. J Vasc Interv Radiol 1997;8:65-70.

12. Do KH, Goo JM, Im JG, Kim KW, Chung JW, Park JH. Systemic arterial supply to the lungs in adults: spiral CT findings. Radiographics 2001;21:387-402.

13. Mal H, Rullon I, Mellot F, Brugiere O, Sleiman C, Menu Y, et al. Immediate and long-term results of bronchial artery embolization for life-threatening hemoptysis. Chest 1999;115:996-1001.

14. Goh PYT, Lin M, Teo N, Wong DES. Embolization for hemoptysis: a six-year review. Cardiovasc Intervent Radiol 2002; $25: 17-25$.

15. Shigemura N, Wan IY, Simon CH, Wong RH, Hsin MKY, Thung HK, et al. Multidisciplinary management of life-threatening massive hemoptysis: a 10-year experience. Ann Thorac Surg 2009;87:849-53.
16. Knott-Craig CJ, Oostuizen JG, Rossouw G, Joubert JR, Barnard PM. Management and prognosis of massive hemoptysis Recent experience with 120 patients. J Thorac Cardiovasc Surg 1993;105:394-7.

17. Valipour A, Kreuzer A, Koller H, Koessler W, Burghuber OC. Bronchoscopy-guided topical hemostatic tamponade therapy for the management of life-threatening hemoptysis. Chest 2005; 127:2113-8.

18. Fartoukh M, Khalil A, Louis L, Carette MF, Bazelly B, Cadranel J, et al. An integrated approach to diagnosis and management of severe haemoptysis in patients admitted to the intensive care unit: a case series from a referral centre. Respir Res 2007;8:11.

19. Sedghi GN, Adimi NP, Miri M, Malek MM, Pejhan S. Massive hemoptysis: diagnostic approach, risk factors and outcome in six years experience. NAFAS J 2015;2:41-7.

20. Davidson K, Shojaee S. Managing massive hemoptysis. Chest 2020;157:77-88

21. Tsoumakidou M, Chrysofakis G, Tsiligianni I, Maltezakis G, Siafakas NM, Tzanakis N. A prospective analysis of 184 hemoptysis cases-diagnostic impact of chest X-ray, computed tomography, bronchoscopy. Respiration 2006;73:808-14.

22. Mondoni M, Carlucci P, Job S, Parazzini EM, Cipolla G, Pagani M, et al. Observational, multicentre study on the epidemiology of haemoptysis. Eur Respir J 2018;51:1701813.

23. Hsiao EI, Kirsch CM, Kagawa FT, Wehner JH, Jensen WA, Baxter RB. Utility of fiberoptic bronchoscopy before bronchial artery embolization for massive hemoptysis. Am J Roentgenol 2001;177:861-7.

24. Abal AT, Nair PC, Cherian J. Haemoptysis: aetiology, evaluation and outcome - a prospective study in a third-world country. Respir Med 2001;95:548-52.

25. Keshmiri MS, Sharifkashani B, Serati A, Seyedi SR, Naghashzadeh F, Salehi S, et al. Superselective angioembolisation: A report of two rare cases of hemoptysis. Int J Cardiovasc Pract 2019;4:19-21.

26. Fruchter O, Schneer S, Rusanov V, Belenky A, Kramer MR. Bronchial artery embolization for massive hemoptysis: longterm follow-up. Asian Cardiovasc Thorac Ann 2015;23:55-60.

27. Seyyedi SR, Sadeghipour P, Sadr M, Shafe O, Moosavi J, Aloosh $\mathrm{O}$, et al. Outcomes and complications of bronchial angioembolization in patients with massive hemoptysis. Tanaffos 2019;18:310-4.

28. Jougon J, Ballester M, Delcambre F, Mac Bride T, Valat P, Gomez F, et al. Massive hemoptysis: what place for medical and surgical treatment. Eur J Cardio-Thoracic Surg 2002;22:345-51.

29. Springer D, Cofta S, Juszkat R, Żabicki B, GoździkSpychalska J, Nowicka A, et al. The effectiveness of bronchial artery embolisation in patients with haemoptysis. Adv Respir Med 2018;86:220-6.

Received for publication: 26 September 2020. Accepted for publication: 30 November 2020.

This work is licensed under a Creative Commons Attribution-NonCommercial 4.0 International License (CC BY-NC 4.0).

(C) Copyright: the Author(s), 2020

Licensee PAGEPress, Italy

Multidisciplinary Respiratory Medicine 2020; 15:723

doi:10.4081/mrm.2020.723 